\title{
O CRESCIMENTO DE DUAS ESPÉCIES FLORESTAIS PIONEIRAS, PAU-DE-BALSA (Ochroma lagopus Sw.) E CAROBA (Jacaranda copaia D. Don), USADAS PARA RECUPERAÇÃO DE ÁREAS DEGRADADAS PELA AGRICULTURA NA AMAZÔNIA CENTRAL, BRASIL.
}

\author{
Antenor Pereira BARBOSA ${ }^{1}$, Moacir Alberto Assis CAMPOS ${ }^{1}$, Paulo de Tarso \\ Barbosa SAMPAIO', Shozo NAKAMURA ${ }^{2}$, Cláudio de Queiroz Blair GONÇALVES'.
}

\begin{abstract}
RESUMO - O objetivo deste trabalho foi estudar o crescimento das espécies florestais pioneiras pau-de-balsa (Ochroma lagopus Sw.) e caroba (Jacaranda copaia D. Don) para a recuperação de áreas degradadas pela agricultura. Na área, situada no km 120 da BR-174, tinha sido plantado mandioca e banana e abandonada há 8 anos, formando uma capoeira de porte baixo e rala. $\mathrm{O}$ experimento foi instalado em maio/98, com e sem gradagem da área. O espaçamento foi de $3 \times 3 \mathrm{~m}$, em covas de $20 \mathrm{~cm}$ (diâmetro) x $30 \mathrm{~cm}$ (profundidade), com adubação de $150 \mathrm{~g} /$ cova de NPK (416-8) e calcário dolomítico na proporção de 3:1. Para a avaliação do crescimento, foram medidas a altura e o diâmetro das plantas aos 2 meses (julho/98) e a cada ano aproximadamente (junho/99, setembro/00 e maio/01). Os dados foram analisados através do delineamento inteiramente casualisado. A sobrevivência do pau-de-balsa foi maior em área gradeada $(97,1 \%)$ do que em area não gradeada (92,5\%), após o primeiro ano do plantio; da caroba, foi cerca de $90 \%$ e sem diferenças entre as areas. A altura e diâmetro do pau-de-balsa, foram maiores em área gradeada, a partir do primeiro ano, chegando no terceiro ano a $11,85 \mathrm{~m}$ de altura e $11,42 \mathrm{~cm}$ de diâmetro. Na caroba, a diferença ocorreu a partir do segundo ano e no terceiro chegou a $8,37 \mathrm{~m}$ de altura e $11,18 \mathrm{~cm}$ de diâmetro. Além de outros fatores inerentes às espécies, o solo mais friável das áreas gradeadas, possibilitou um maior crescimento em altura e diâmetro das duas espécies estudadas.
\end{abstract}

Palavras-chave: Ochroma lagopus, Jacaranda copaia, áreas degradadas, Amazônia, Brasil.

\section{Growth of two forest pioneer species, pau-de-balsa (Ochroma lagopus Sw.) e caroba (Jacaranda copaia D. Don), used for rehabilitation of degraded areas from agriculture in Central Amazon, Brazil.}

\begin{abstract}
The objective of experiment was study the growth of pioneer forest species paude-balsa (Ochroma lagopus) and caroba (Jacaranda copaia) to rehabilitate degraded areas from agriculture. The experiment carried out at Br-174, $\mathrm{km} \mathrm{120.} \mathrm{After} \mathrm{the} \mathrm{use} \mathrm{for} \mathrm{cassava} \mathrm{and} \mathrm{banana}$ plantations the area was abandoned for 8 years. The secondary forest that took place was of low height and sparse trees. The experiment was installed at may/98 and composed by harrowed and no harrowed areas. The seedlings were planted in $3 \times 3 \mathrm{~m}$ spacing, in $20 \mathrm{~cm}$ diameter by $30 \mathrm{~cm}$ deep holes, with $150 \mathrm{~g}$ of fertilizer NPK (4-16-8) and dolomite limestone in the proportion 3:1. To evaluation the growth were measured the height and diameter in july/98 and each year, approximately (June/99, September/00 and May/01). The data were obtained from planted species comparing harrowed and no harrowed areas in a full random design. The survival of pau-debalsa, was greater in harrowed area $(97,1 \%)$ in relation to no harrowed area $(92,5 \%)$ after one year of planting; and the survival of caroba, was $90 \%$ after one year of planting and showed no

\footnotetext{
${ }^{1}$ Instituto Nacional de Pesquisas da Amazônia/INPA - Coordenação de Pesquisas em Silvicultura Tropical (CPST). Cx. Postal 478, CEP 69.083-000, Manaus, AM, Brasil.
}

${ }^{2}$ Pesquisador do FFPRI e perito de longo prazo no Projeto JACARANDA (INPA/JICA)
\end{abstract}


difference when compared the harrowed and no harrowed areas. The height and diameter of paude-balsa were greater in harrowed area after one year of planting, reaching $11.85 \mathrm{~m}$ and $11.42 \mathrm{~cm}$, respectively. The differences occurred in caroba trees after the second year of planting, and reached, in the third year, $8.37 \mathrm{~m}$ of high and $11,18 \mathrm{~cm}$ in diameter. Besides other factors inherent to the species, the more friable soil of the harrowed area, facilitated higher growth to two studied species.

Key-words: Ochroma lagopus, Jacaranda copaia, degraded area, Amazon, Brazil.

\section{INTRODUÇÃO}

Os desmatamentos realizados na Amazônia para que a terra seja usada para agricultura ou pecuária, continuam sendo comuns na região. Na maioria das vezes, são seguidos de queimadas, sem opções ecológicas e economicamente seguras para ocupação futura. Esta prática e a falta de manejo adequado das culturas e do solo, ocasionam uma degradação múltipla como a erosão física, química e microbiológica, levando a uma drástica redução da capacidade de reabilitação natural da área.

Entre os critérios propostos para a implantação ou recomposição de matas e/ou recuperação de áreas degradadas existe o de distribuição aleatória das mudas das diferentes espécies, o de distribuição indicada por estudos fitossociológicos de matas naturais adjacentes e o de distribuição baseada na combinação de grupos de espécies de diferentes estádios de sucessão secundária (Botelho et al., 1995).

O sistema baseado na sucessão florestal é o que vem obtendo mais êxito, porque favorece a rápida cobertura do solo e garante a auto-renovação da floresta. As estratégias diferenciáveis das espécies dentro da dinâmica das florestas, constituem um conceito chave para a compreensão do processo de sucessão, tanto a nível de regeneração de grandes áreas após a eliminação da floresta, como também a nível local na chamada dinâmica de clareiras, que ocorre durante qualquer estádio sucessional (Botelho et al., 1995). Segundo Carpanezzi et al. (1990), o reflorestamento pioneiro da área degradada é a ação inicial no método sucessional e cumpre outros objetivos importantes, como o estético e o hidrológico.

A silvicultura pró-sucessão é integrante do método sucessional e difere da silvicultura comercial pelo paradigma das espécies, entre outros aspectos. (Kageyama et al 1989).
$\mathrm{Na}$ definição das espécies a serem plantadas e do esquema de distribuição, algumas questões devem ser consideradas: quantas e quais as espécies a serem utilizadas, quantos indivíduos de cada espécie e qual o melhor arranjo para a distribuição das espécies? As espécies devem ser selecionadas, preferencialmente, entre as que ocorrem na mesma região climática e de solo do local do plantio, devido a interação que ocorre entre as espécies e o ambiente (Botelho et al., 1995).

As espécies pioneiras selecionadas foram pau-de-balsa (Ochroma lagopus Sw.) e caroba (Jacaranda copaia D. Don). Segundo Vasquez-Yanes (1974), o pau-de-balsa é uma espécie característica das primeiras etapas de sucessão secundária em zonas tropicais úmidas, e que temperaturas altas, provocadas pelo calor seco e úmido, produzem aumento de percentagem de germinação. Ochroma lagopus ocorre na faixa de $19^{\circ} \mathrm{N}$ e $20^{\circ} \mathrm{S}$ em regiões tropicais das Américas do Sul e Central e em altitudes de 0 a $1000 \mathrm{~m}$. A madeira é muito leve $\left(0,20\right.$ a $\left.0,35 \mathrm{~g} / \mathrm{cm}^{3}\right)$ e pode ser utilizada para fabricação de brinquedos, forros de teto, material isolante, para caixas de embalagem, construção aeronáutica e material flutuante (Loureiro et al 1979), apresentando um alto potencial de uso na fabricação de chapas de cimento-madeira para divisórias, móveis, pisos, etc. com madeira de plantas com cerca de um ano de idade.

A caroba é uma árvore grande, de crescimento rápido com flores azul-violáceas; que ocorre em toda a Amazônia e habita matas e capoeiras velhas de terra firme. A madeira é leve, fácil de trabalhar, empregada na fabricação de brinquedos, marcenaria, papel, balsas e trabalhos de interior (Loureiro et al., 1979). Para Sampaio et al. (1989), pelo rápido crescimento e boa forma do fuste, a espécie tem um bom potencial para programas de reflorestamento na região amazônica. 
Este trabalho teve como objetivo a análise do crescimento inicial de espécies florestais pioneiras plantadas em áreas degradadas pela agricultura, no Município de Presidente Figueiredo, Estado do Amazonas.

\section{MATERIAIS E MÉTODOS}

A área utilizada para a instalação do experimento está localizada no Ramal Boa Esperança, às margens da BR-174, km 120 no Município de Presidente Figueiredo no Estado do Amazonas e pertence à A. Teixeira Mendes LTDA. Segundo informações do proprietário, a área foi usada para o plantio de mandioca e banana por vários anos e abandonada a aproximadamente 8 anos. Nesse período, desenvolveu-se naturalmente uma capoeira rala e baixa expressando uma aparente necessidade de intervenção para um melhor crescimento da vegetação. Os solos da área são Latossolos amarelos, muito ácidos com $\mathrm{pH}$ entre 4,0 e 4,3. O clima da região está classificado como 'Afi' pelo sistema de Köppen (Clima Tropical Chuvoso) e caracteriza-se por apresentar temperatura média do mês mais frio nunca inferior a $18^{\circ} \mathrm{C}$. A precipitação normal é de $2526 \mathrm{~mm}$, no período de 1971 a 1997 . A temperatura média anual é de $25,8^{\circ} \mathrm{C}$, com temperatura média mensal máxima de $31,4^{\circ} \mathrm{C}$ e mínima de $22,2^{\circ} \mathrm{C}$. A umidade relativa do ar é de cerca de $86 \%$ (EMBRAPA, 1998).

Após a retirada da vegetação natural com trator de esteira, o experimento foi instalado em maio/98 e foi composto por dois preparos de área: com e sem gradagem, onde foram plantadas, em cada uma delas, mudas das espécies pioneiras pau-de-balsa (Ochroma lagopus Sw.) e caroba (Jacaranda copaia D.
Don). O plantio foi feito em covas com $20 \mathrm{~cm}$ de diâmetro x $30 \mathrm{~cm}$ de profundidade, espaçadas de $3 \times 3 \mathrm{~m}$, com adubação mineral de $150 \mathrm{~g} /$ cova de NPK (4-16-8) e calcário dolomítico na proporção de $3: 1$, misturados na terra da cova. Na falta de informações sobre as exigências nutricionais das espécies deste estudo, adotou-se a referida adubação como básica, uma vez que o adubo utilizado, além dos macronutrientes, também continham micronutrientes. Para avaliar o efeito da gradagem do solo, foram medidas a altura total e o diâmetro do colo ou DAP das plantas aos 2 meses (julho/98) e a cada ano aproximadamente (junho/99, setembro/00 e maio/01). Os dados foram analisados através do delineamento inteiramente casualisado com 15 repetições, mantida uma bordadura simples. Cada repetição foi composta pela média de uma linha com 27 plantas.

\section{RESULTADOS}

\section{Sobrevivência do pau-de-balsa (Ochroma lagopus Sw.) e caroba (Jacaranda copaia D. Don).}

A sobrevivência das mudas de pau-debalsa, em todos os períodos das observações, foi maior em área gradeada com 97,1\% (Tabela 1). A maior percentagem de sobrevivência a partir das observações de junho/99, ocorreu porque foi feito o replantio das mudas mortas após a avaliação de julho/98. Na caroba, nesse período, não ocorreu diferença na sobrevivência das mudas plantadas nas áreas com e sem gradagem. Apesar do replantio, após julho/ 98, a sobrevivência continuou diminuindo, no entanto permaneceu em cerca de $90 \%$ (Tabela 1).

Tabela 1 - Percentagens da sobrevivência das mudas de pau-de-balsa (Ochroma lagopus Sw.) e caroba (Jacaranda copaia D. Don) plantadas para recuperação de áreas degradadas pela agricultura, no Município de Presidente Figueiredo (AM)*.

\begin{tabular}{ccccc}
\hline PERÍODO & \multicolumn{3}{c}{ SOBREVIVÊNCIA DAS ESPÉCIES PIONEIRAS (\%) } \\
\cline { 2 - 5 } DE & \multicolumn{2}{c}{ pau-de-balsa } & \multicolumn{2}{c}{ caroba } \\
\cline { 2 - 5 } MEDIÇÃO & Área gradeada & Não gradeada & Área gradeada & Não gradeada \\
\hline Julho/98 & $84,3 \mathrm{a}$ & $54,9 \mathrm{~b}$ & $96,4 \mathrm{a}$ & $91,2 \mathrm{~b}$ \\
Junho/99 & $97,1 \mathrm{a}$ & $92,5 \mathrm{~b}$ & $90,3 \mathrm{~ns}$ & $88,8 \mathrm{~ns}$ \\
Setembro/00 & $97,1 \mathrm{a}$ & $92,5 \mathrm{~b}$ & $90,1 \mathrm{~ns}$ & $88,5 \mathrm{~ns}$ \\
Maio/01 & $97,1 \mathrm{a}$ & $92,5 \mathrm{~b}$ & $89,6 \mathrm{~ns}$ & $88,5 \mathrm{~ns}$ \\
\hline
\end{tabular}

* Médias seguidas pelas mesmas letras na linha, em cada espécie, não diferem significativamente entre si pelo teste de Tukey ao nível de $5 \%$ de probabilidade. 


\section{Crescimento em altura do pau- de-balsa (Ochroma lagopus Sw.) e caroba (Jacaranda copaia D. Don).}

A altura do pau-de-balsa nos dois primeiros meses após o plantio, não apresentou diferenças entre os dois tipos de preparo de solo (Tabela 2). Em área gradeada, as mudas estavam com 1,36 m e na área não gradeada com 1,22 m. Um ano depois, em junho/99, as mudas plantadas em solos gradeados tinham a altura média de $8,13 \mathrm{~m}$ e significativamente maior do que aquela da área não gradeada, que estava com 5,95 m. O crescimento das mudas nos dois anos seguintes (setembro/00 e maio/ $01)$, na área gradeada, continuou maior $(11,36$ $\mathrm{m}$ e $11,85 \mathrm{~m}$ ) quando comparado com o da área não gradeada $(8,94 \mathrm{~m}$ e 9,62 m), respectivamente.

Os dados mostram que a resposta da gradagem do solo no crescimento em altura, ocorreu, aproximadamente, um ano após o plantio, quando o pau-de-balsa chegou a crescer cerca de $6,8 \mathrm{~m} /$ ano em média. A partir deste período, a taxa de crescimento drasticamente diminuiu para 3,2 $\mathrm{m}$ e $0,5 \mathrm{~m}$ entre os dois anos seguintes setembro/00 e maio/01, respectivamente.

A altura das mudas de caroba, permaneceu sem diferenças significativas até o final do primeiro ano do plantio, quando estavam com 2,37 m e 2,57 m, nas áreas gradeadas e não gradeadas, respectivamente (Tabela 2). Após dois anos, o efeito da gradagem do solo permitiu um maior crescimento em altura, quando as mudas alcançaram 6,36 m. Esse resultado continuou no ano seguinte, quando a altura da caroba chegou a $8,37 \mathrm{~m}$ na área gradeada e 7,01 $\mathrm{m}$ na não gradeada.

O pau-de-balsa, teve uma maior taxa de crescimento durante o primeiro ano $(6,8 \mathrm{~m})$, enquanto que o crescimento na caroba foi maior no intervalo do primeiro para o segundo ano (junho/99 a setembro/00) e foi de 3,63 m. No intervalo do segundo para o terceiro ano (setembro/00 a maio/01) a taxa de crescimento em altura da caroba $(2,01 \mathrm{~m})$ foi maior do que a do pau-de-balsa $(0,5 \mathrm{~m})$.

$\mathrm{O}$ crescimento em altura do pau-debalsa plantado em área gradeada foi maior quando comparado com a Ceiba pentandra, também considerada pioneira, que alcançou $8,58 \mathrm{~m}$ de altura aos 4 anos de plantada (Azevedo, 1997). Piña Rodrigues et al. (1997) plantaram 15 espécies pioneiras e, as que tiveram o maior crescimento, foram o vinhático e a aleluia, as quais alcançaram 3,3m aos 14 meses de plantadas. Silva et al. (1997) plantaram várias espécies na região de Urucu, no Estado do Amazonas e encontraram os maiores crescimentos nas espécies pioneiras, com 1,5m de altura após 6 meses do plantio. Jacaranda mimosaefolia, uma espécie do mesmo gênero da caroba, alcançou $2,85 \mathrm{~m}$ de altura aos 16 meses de idade (Barbosa et al., 1997).

$\mathrm{O}$ rápido crescimento do pau-de-balsa, no primeiro ano, pode ser atribuído, à morfologia foliar em desenvolver folhas arredondadas com limbos grandes de mais de $20 \mathrm{~cm}$ de diâmetro e possuir um sistema radicular abundante e de muitas raízes finas. Essas características podem permitir uma maior

Tabela 2 - Altura total (m) das mudas de pau-de-balsa (Ochroma lagopus Sw.) e caroba (Jaca randa copaia $\mathrm{D}$. Don) plantadas para recuperação de áreas degradadas pela agricultura, no Município de Presidente Figueiredo (AM).

\begin{tabular}{ccccc}
\hline \multirow{2}{*}{ PERIODO } & \multicolumn{2}{c}{ ALTURA TOTAL } \\
\cline { 2 - 4 } DE & \multicolumn{2}{c}{ paúde-balsa } & \multicolumn{2}{c}{ caroba } \\
\cline { 2 - 5 } MEDIÇÃO & Área gradeada & Não gradeada & Área gradeada & Não gradeada \\
\hline Julho/98 & $1,36 \mathrm{~ns}$ & $1,22 \mathrm{~ns}$ & $0,32 \mathrm{~ns}$ & $0,35 \mathrm{~ns}$ \\
Junho/99 & $8,13 \mathrm{a}$ & $5,95 \mathrm{~b}$ & $2,73 \mathrm{~ns}$ & $2,57 \mathrm{~ns}$ \\
Setembro/00 & $11,36 \mathrm{a}$ & $8,94 \mathrm{~b}$ & $6,36 \mathrm{a}$ & $4,94 \mathrm{~b}$ \\
Maio/01 & $11,85 \mathrm{a}$ & $9,62 \mathrm{~b}$ & $8,37 \mathrm{a}$ & $7,01 \mathrm{~b}$ \\
\hline
\end{tabular}

* Médias seguidas pelas mesmas letras na linha, em cada espécie, não diferem significativamente entre si pelo teste de Tukey ao nível de $5 \%$ de probabilidade. 
captação da radiação solar e uma maior capacidade de absorção de água e nutrientes do solo, principalmente, nos gradeados que são mais frouxos e aerados. Isto é evidenciado porque a copa das mudas de pau-de-balsa cresceram rapidamente no primeiro ano e depois, se tornaram auto-competitivas por luz, se entrelaçando e ocasionando autosombreamento.

A caroba, diferentemente do pau-debalsa, possui folhas compostas com folíolos pequenos, copa pequena, geralmente sem ramificação nos primeiros 3 anos e com uma redução na taxa de crescimento em altura, um ano mais tarde (Tabela 2). Além dos fatores inerentes à espécie, pode ter ocorrido uma concorrência por água e nutrientes do solo, visto que as copas ainda não se entrelaçavam neste período para ocasionar o autosombreamento.

Os dados mostram a tendência de que a altura da caroba alcance a do pau-de-balsa nos anos seguintes nestas condições de plantio.

\section{Crescimento em diâmetro do pau-de-balsa (Ochroma lagopus Sw.) e caroba (Jacaranda copaia D. Don).}

O crescimento em diâmetro das mudas de pau-de-balsa e caroba, dois meses após o plantio (julho/98), não mostrou diferenças entre as áreas gradeada e não gradeada. A partir do primeiro ano (junho/99), o diâmetro do paude-balsa foi maior em área gradeada, quando comparado com a área não gradeada. No segundo (setembro/00) e terceiro anos (maio/
01), o diâmetro continuou maior em área gradeada e alcançou 11,42 cm. Na caroba, a diferença de crescimento em diâmetro ocorreu a partir do segundo ano do plantio (setembro/ $00)$, chegando no terceiro ano (maio/01) a alcançar $11,18 \mathrm{~cm}$ em área gradeada e $10,13 \mathrm{~cm}$ em área não gradeada, valores semelhantes aos alcançados pelo pau-de-balsa (Tabela 3).

Hamzah et al. (1995), usaram diversas espécies nativas da Malasia para a recuperação de áreas degradadas e o máximo alcançado foi de $1,74 \mathrm{~cm}$ no período de 1 ano após o plantio. No primeiro ano, o pau-de-balsa tinha o diâmetro de $9,19 \mathrm{~cm}$ e a caroba de $7,78 \mathrm{~cm}$ em área gradeada. No primeiro ano, o pau-debalsa cresceu $6,01 \mathrm{~cm}$ de diâmetro e a caroba de $6,61 \mathrm{~cm}$ em área gradeada. No segundo ano, o diâmetro aumentou 2,06 cm no pau-de-balsa e $1,47 \mathrm{~cm}$ na caroba. E, no terceiro ano, o aumento foi de $0,17 \mathrm{~cm}$ no pau-de-balsa e de $1,93 \mathrm{~cm}$ na caroba (Tabela 3).

De modo semelhante à altura, o diâmetro da caroba tendeu a ficar maior do que do paude-balsa no final do terceiro ano. Os diferentes fatores de forma e tamanho da copa, esgalhamento e desenvolvimento do sistema radicular influenciaram o crescimento das espécies produzindo diferentes condições de concorrência por luz, água e nutrientes do solo.

Além dos outros fatores inerentes às espécies, o solo mais solto resultante da gradagem da área, aumentou o crescimento em altura e diâmetro nos três primeiros anos após o plantio com diferentes taxas de crescimento.

Tabela 3 - Diâmetros (cm) das mudas de pau-de-balsa (Ochroma lagopus Sw.) e caroba (Jaca randa copaia $\mathrm{D}$. Don) plantadas para recuperação de áreas degradadas pela agricultura,

\begin{tabular}{ccccc}
\hline \multirow{2}{*}{ PERÍODO } & \multicolumn{2}{c}{ DIÂMETRO MÉDIO DAS ESPÉCIES PIONEIRAS $(\mathrm{cm})^{*}$} \\
\cline { 2 - 5 } DE & \multicolumn{2}{c}{ pau-de-balsa } & \multicolumn{2}{c}{ caroba } \\
\cline { 2 - 5 } MEDIÇÃO & Área gradeada & Não gradeada & Área gradeada & Não gradeada \\
\hline Julho/98 & $3,18 \mathrm{~ns}$ & $2,95 \mathrm{~ns}$ & $1,17 \mathrm{~ns}$ & $1,25 \mathrm{~ns}$ \\
Junho/99 & $9,19 \mathrm{a}$ & $7,28 \mathrm{~b}$ & $7,78 \mathrm{~ns}$ & $7,66 \mathrm{~ns}$ \\
Setembro/00 & $11,25 \mathrm{a}$ & $9,04 \mathrm{~b}$ & $9,25 \mathrm{a}$ & $8,42 \mathrm{~b}$ \\
Maio/01 & $11,42 \mathrm{a}$ & $9,64 \mathrm{~b}$ & $11,18 \mathrm{a}$ & $10,13 \mathrm{~b}$ \\
\hline
\end{tabular}

* Médias seguidas pelas mesmas letras na linha, em cada espécie, não diferem significativamente entre si pelo teste de Tukey ao nível de $5 \%$ de probabilidade. 


\section{CONCLUSÕES}

As sobrevivências do pau-de-balsa e da caroba, foram altas com valores de 97,1\% e cerca de $90 \%$, respectivamente. A gradagem da área aumentou a sobrevivência do pau-de-balsa e, na caroba, não mostrou diferença significativa.

A maior taxa de crescimento do paude-balsa ocorreu no primeiro ano após o plantio em área gradeada e, na caroba, isto aconteceu no segundo ano também em área gradeada.

A gradagem do solo aumentou o crescimento em altura e diâmetro do pau-debalsa e da caroba nos três primeiros anos após o plantio.

Para se detectar os fatores que podem ter influenciado a drástica redução da taxa de crescimento do pau-de-balsa após o primeiro ano, sugere-se um monitoramento trimestral das condições nutricionais do solo, de luminosidade para avaliar o auto-sombreamento associado a diferentes níveis de desbastes, quando as copas iniciarem a se entrelaçar em plantios com espaçamento de 3 x 3 metros.

\section{BIBLIOGRAFIA CITADA}

Azevedo, C. P. 1997. Recuperação e aproveitamento de áreas abandonadas el ou degradadas através de plantios florestais. EMBRAPA-CPAA. Manaus Am. $14 \mathrm{p}$.

Barbosa, L.M.; GISLER, C. V.T.; ASPERTI, L.M. 1997. Desenvolvimento inicial de oito espécies arbóreas em dois modelos de reflorestamento implantados em área de mata ciliar degradada em Santa Cruz das Palmeiras, SP. In: III SINRAD. SOBRADE, U.F.V., Ouro Preto, MG. p. 437-445.

Botelho, S. A; Davide, A.C.; Prado, N.J.S.; Fonseca, E.M.B. 1995. Implantação de mata ciliar. Companhia Energética de Minas Gerais. Belo Horizonte: CEMIG. Lavras/UFLA. 28p.

Carpanezzi, A. A.; Costa, L. G. S.; Kageiama, P. Y.; Castro, C. F. de A. 1990. Espécies pioneiras para recuperação de áreas degradadas: A observação de laboratórios naturais. In: $6^{\circ}$ Congresso Florestal Brasileiro. Campos do Jordão (SP). p. 216-222.
EMBRAPA. 1998. Boletim Agrometeorológico. Manaus: EMBRAPA-CPAA, Anual.

Hamzah, M. Z.; Bojo, Othman; Taha, A.H.M.; Alias, M.A.; Majid, N.M.; Malek, I.A.A. 1995. Rehabilitation of degraded tropical forest area in Sarawak using indigenous tree species-The Bintulu experience. EcoHabitat, 2(1): 11-17.

Kageyama, P. Y.; Castro, C. F. A.; \& Carpanezzi, A. A. 1989. Implantação de matas ciliares: estratégias para auxiliar a sucessão secundária. In: Barbosa, L. M. (Coord) Simpósio sobre mata ciliar. São Paulo. Anais Fundação Cargill, Campinas 872p.

Loureiro, A.A.; Silva, M.F. \& Alencar, J.C. 1979. Essências madeireiras da Amazônia. Vol. II. INPA, Manaus, AM, Brasil. 187p.

Piña-Rodrigues, F.C.M.; Lopes, L.; Bloomfield, V.K. 1997. Análise do desenvolvimento de espécies arbóreas da mata Atlântica em plantio adensado para a revegetação de áreas degradadas em encostas, no entorno do parque Estadual do Desengano (RJ). In: III SINRAD. SOBRADE, U.F.V., Ouro Preto, MG.p. 283-291.

Sampaio, P.T.B.; Barbosa, A.P.; Fernandes, N.P. 1989. Ensaio de espaçamento com caroba - Jacaranda copaia (AUBL.) D. DON. BIGNONIACEAE. Acta Amazonica, 19 (único):383-389.

Silva, K. E.; Tucci, C.A.F.; Almeida, N.O.A.; Lima, H.N. 1997. Revegetação de clareiras na Base Petrolífera do Rio Urucu-Am. Avaliação preliminar. In: III. SINRAD. SOBRADE, U.F.V., Ouro Preto, MG. p. 270-275.

Vasquez-Yanes, C. 1974. Studies on the germination of seeds of Ochroma lagopus Sw. Turrialba, 24(2): 176-179.

\section{Recebido: 27/08/2001}

Aceito: 09/10/2002 